\title{
Erratum to: Mathematical Modeling and Numerical Algorithms for Simulation of Oil Pollution
}

\author{
Quang A. Dang • Matthias Ehrhardt • Gia Lich Tran • \\ Duc Le
}

Published online: 27 May 2012

(C) Springer Science+Business Media B.V. 2012

Erratum to: Environ Model Assess (2012) 17:275-288 DOI 10.1007/s10666-011-9291-1

Unfortunately, equation 3 was incorrect. The corrected version of equation is given below.

$\nabla \cdot \mathbf{U}=\frac{\partial u}{\partial x}+\frac{\partial v}{\partial y}=0$

The online version of the original article can be found at http://dx.doi.org/ 10.1007/s10666-011-9291-1.

Q. A. Dang

Institute of Information Technology,

18 Hoang Quoc Viet,

Ha noi, Vietnam

e-mail: dangqa@ioit.ac.vn

M. Ehrhardt $(\bowtie)$

Lehrstuhl für Angewandte Mathematik und Numerische Analysis,

Fachbereich C Mathematik und Naturwissenschaften,

Bergische Universität Wuppertal,

Gaußstrasse 20,

42119 Wuppertal, Germany

e-mail: ehrhardt@math.uni-wuppertal.de

G. L. Tran

Institute of Mathematics,

18 Hoang Quoc Viet,

Ha noi, Vietnam

e-mail: tranglich@math.ac.vn

D. Le

Buro of Hydrology and Meteorology,

Dang Thai Than,

Ha noi, Vietnam

e-mail: Leduc@nchmf.gov.vn 